\title{
Multiobjective Optimization Applied to Maintenance Policy for Electrical Networks
}

\author{
Patrik Hilber, Graduate Student Member, IEEE, Vladimiro Miranda Fellow, IEEE, Manuel Matos, \\ Member, IEEE, and Lina Bertling, Member, IEEE
}

\begin{abstract}
This paper propose an approach to multi-objective maintenance policy definition for electrical networks. Maximum asset performance is one of the major goals for electric power system managers. To reach this goal, minimal life cycle cost and maintenance optimization becomes crucial, while meeting demands from customers and regulators. This necessitates the determination of the optimal balance between preventive and corrective maintenance in order to obtain the lowest total cost.

The approach of this paper is to study the problem of balance between preventive and corrective maintenance as a multiobjective optimization problem, where we have the customer interruptions on one hand and on the other hand the maintenance budget of the network operator. The problem is solved with meta-heuristics developed for the specific problem, as well as with an Evolutionary Particle Swarm Optimization algorithm.

The maintenance optimization is applied in a case study to an urban distribution system in Stockholm, Sweden. Despite a general decreased level of maintenance (lower total maintenance cost) a better network performance can be given to the customers. This is achieved by focusing the preventive maintenance on components with a high potential for improvements. Beside this, the paper constitutes a display of the value in introducing more maintenance alternatives for every component and to choose the right level of maintenance for the components with respect to network performance.
\end{abstract}

Index Terms-Asset management, Component reliability importance, Maintenance, Multiobjective optimization, Power distribution systems.

\section{INTRODUCTION}

$\mathrm{M}$ AXIMUM asset performance is one of the major goals for electric power system managers. To reach this goal minimal life cycle cost and maintenance optimization becomes crucial while meeting demands from customers and regulators. One of the fundamental objectives is therefore to relate maintenance to system reliability performance in an efficient and effective way. This is the aim of several maintenance methods such as the Reliability Centered

Manuscript received June 8, 2007. This work was in part supported by the Swedish research foundation Elektra.

P. Hilber and L. Bertling are with KTH (Royal Institute of Technology), School of Electrical Engineering, Stockholm, Sweden. V. Miranda and M. Matos are with the Power Systems Unit, INESC Porto, Porto, Portugal and also with Faculty of Engineering, Univ. of Porto, Portugal. Corresponding author: P. Hilber, phone: +46 879077 72; e-mail: hilber@kth.se.
Maintenance method [1] and further developed methods such as the Reliability Centered Asset Management [2]. In this context it becomes crucial to find the solution to the problem of optimal balance between corrective and preventive maintenance (the maintenance problem). In the literature a number of methods exist, e.g. [3], [4] and [5], that focus on capturing the optimal level of maintenance with respect to a specific objective, such as minimizing a specific interruption index while meeting a budget constraint. This paper takes the concept of these methods further by applying the methods of multiobjective optimization to the maintenance optimization. This is done in a similar approach as in the distribution system development planning described in [6], where a multiobjective approached is utilized. The difference is that the proposed method is aimed at maintenance instead of planning. Furthermore, the presented method is developed in order to deliver optimal solutions for computational intense reliability calculations that are based on simulations. This is an attribute that allows for detailed modeling of the studied network. The multiobjective approach puts the customer interruption on one hand and the maintenance budget of the distribution system operator (DSO) on the other. Hence, the proposed method provides a span of optimal solutions that the decision maker can chose between. Each with different expected outcomes for maintenance budget and customers.

\section{OPTIMIZATION PROBLEM, METHOD}

The approach of this paper is a maintenance policy optimization, which results in a number of interesting solutions that the decision maker can chose between. The solutions are developed by utilizing component reliability importance indices, derived from Monte Carlo reliability simulations. The method is divided into five essential concepts:
A. Reliability Monte Carlo simulations.
$B$. Component reliability importance indices.
C. Multiobjective approach.
$D$. Optimization heuristics.
E. Selection of optimum (results).

The merit of this paper lies within the combination of these concepts, below presented in their respective subsection.

\section{A. Event driven Monte Carlo Simulation}

The Monte Carlo simulation method is based on an event driven approach. I.e. a simulation with variable time steps, 
where the time between significant events is identified. In this context significant events are state changes of components. Minimal cut-sets are used to calculate the system behavior as a result of component status. In the current setup the simulation runs for a specified number of cycles. At the simulation start we assume that all components are functional. The next step is to generate the time to failure for every component. Time to failure is based on the component's failure rate, which in turn is based on component parameters, e.g. level of maintenance and inherent failure rate. We then move forward in time to the first event, in our case a failed component. The changed status of the failed component is then recorded along with the effects of the failure. The list of coming events is consequently updated with the generated repair time for the failed component. This is followed by the identification of the next event in the simulation. Probably being the repair of the failed component but not impossibly an occurrence of another failed component and consequences of that (e.g. a $2^{\text {nd }}$ order minimal cut-set failure). By recording the effects of the different system states and their durations we obtain reliability data, such as the utilized component importance index and more traditional reliability data such as customer interruption costs and SAIFI and SAIDI [7]. The simulation approach and its implementation into the reliability analysis tool RADPOW is presented in [8].

Simulations compared to analytical calculations make the optimization more complicated. This since Monte Carlo simulations to a certain degree delivers stochastic output and in general can be said to require more calculation-time per reliability calculation. The simulations do, however, bring us the possibility of implementing more complicated model behaviors.

\section{B. Component reliability importance index}

Component reliability importance indices provide the connection between component and system reliability performance [9]. The component reliability importance index used in this paper, $I^{M}$ [10], corresponds to the expected total customer interruption cost caused by the studied component over a specific time interval (usually one year). The index, which was developed for calculation by simulation [10], is calculated by accumulating the total interruption cost caused by every interruption to the finally causing component over all simulated years. The accumulated cost for every component is then divided with the total simulation time in order to get an expected interruption cost per time unit. The index is defined as follows (1):

$I_{i}^{M}=\frac{K_{i}}{T} \quad[€ / \mathrm{yr}]$

where $K_{i}$ is the total accumulated customer interruption cost over the total simulated time $T$ for component $i$. The index gives an indication of which components should be prioritized for preventive maintenance actions (or in some cases redesigns of the structures that result in the high value of $I^{M}$ ). Moreover, $I^{M}$ gives information on components that do not cause much interruption cost for the network. It might for example be beneficial to reduce preventive maintenance for these components. However, to reach the best possible solution, this information needs to be adequately combined with knowledge about available maintenance actions, their costs and estimated effects.

\section{Multiobjective approach to the optimization problem}

The studied problem, finding the optimal balance of preventive and corrective maintenance, is approached as a multicriteria/multiobjective optimization problem. On one hand we have the customers requirements on power delivery and on the other hand we have the maintenance cost for the DSO. In this study we have chosen to use the total customer interruption cost as the measure of network performance from the customer perspective. The maintenance costs are closely related to the analyzed network, its components, structure and available resources.

It is possible to extend the multiobjective approach by studying every load point's availability as an individual objective. Some cases might for example call for pure pareto improvements [11], where all customers are viewed separately, i.e. improvements that reduce costs or at least keep costs at current level for all involved parties. To study all customers independently while requiring pareto improvements narrows down the feasible solution space. Furthermore, with more objectives the solution space fast becomes difficult to grasp with increasing number of load points.

It is interesting to note that the two objectives (customer interruption cost and cost of maintenance) not entirely points the solution into two different directions since the cost of corrective maintenance to a certain degree correlates with the customers inconvenience.

\section{Heuristic optimization approach: AGEBOM - Approximate Gradient Evaluation Bi-criteria Optimization Method}

The proposed optimization is based on an aggregated auxiliary objective function that incorporates the two objectives, the customer interruption cost and the maintenance cost of the network (both corrective and preventive).

\section{1) Objective function}

A scaling between the customer interruption cost and the maintenance cost of the network is introduced. This scaling is varied in order to obtain a number of non-dominated solutions with specific tradeoff between customers and DSO. The objective function of the optimization is presented in (2).

$\min s^{*} C^{I C}+C^{C M}+C^{P M} \quad[€ / \mathrm{yr}]$

where $C^{I C}[€ / \mathrm{yr}]$ is the expected yearly system customer interruption cost, $C^{C M}[€ / \mathrm{yr}]$ the cost of corrective maintenance, $C^{P M}[€ / \mathrm{yr}]$ the cost of preventive maintenance and $s$ is the scale factor (tradeoff). The unit of the scale factor, $s$, becomes DSO money per unit of customer money. The scale factor constitutes a translation of the expected customer interruption costs into terms of DSO costs. $C^{C C}$ is obtained from simulations [10] and depends on the maintenance strategy. The values of $C^{C M}$ and $C^{P M}$ are based on the maintenance strategy; see the case study for an example. The 
three different costs in the objective function depend on the network and its components. That is components failure rates, repair times and network structure and operation. In addition the simulation delivers the component reliability importance index, $I^{M}[€ / \mathrm{yr}]$, for every component [10]. The index $I^{M}$ corresponds to the average yearly customer interruption cost caused by the specific component. How $C^{P M}$ and $C^{C M}$ are calculated depends largely on the studied network; for this paper these costs are presented in the case study. $C^{I C}$ is calculated according to (3) [12].

$$
C^{C C}=\sum_{L} \lambda_{L}\left(k_{L} P_{L}+c_{L} P_{L} r_{L}\right) \quad[€ / \mathrm{yr}]
$$

where $C^{I C}$ is the total expected yearly customer interruption cost for the system, $P_{L}[\mathrm{~kW}]$ average power, $\lambda_{L}[\mathrm{f} / \mathrm{yr}]$ and $r_{L}$ $[\mathrm{h} / \mathrm{f}]$ are reliability indices for every load point $L$, and $k_{L}[€ / \mathrm{f}$, $\mathrm{kW}]$ and $c_{L}[€ / \mathrm{kWh}]$ are cost constants representing the customer types and composition for every load point $L$.

\section{2) Workflow of optimization}

Since the reliability calculations of customer interruption costs and component importance are derived from simulations, the optimization approach is pushed toward a method that requires few calls on calculation of objective function and other outputs. Another aspect is that the simulations constitutes a "black box" that an optimization routine can not see through. However, the concept of component reliability importance indices allows for a certain degree of visibility into the "black box".

It is assumed that the caused interruption cost is linearly dependant on the failure rate of the component, when no other data is changed. I.e. assuming that a relative change in failure rate result in the same relative change in customer interruption cost. Given maintenance actions and estimates on failure rate changes and maintenance cost/savings caused by these, a costbenefit ratio can be developed. That is a ratio between the change in interruption cost and the cost/savings of the investigated action. By doing this for all available actions for all components the available actions can be ranked.

The optimization, which can be described as a steepest descent method, commence with a leap. The leap introduces the best cost-benefit ratio actions for each component into the solution. This is done despite violating the assumption above. The leap is followed by a stepwise approach that does not violate the above assumption. I.e. all available maintenance actions are evaluated, but only the most profitable one is selected, given that it is expected to result in a better objective function. The optimization is illustrated in Fig. 1.

The start condition for the optimization is that all components are at their initial (current) state. Then a reliability calculation (simulation) is performed. The index $I^{M}$ which is an output from the simulation is used in order to estimate the impacts on the objective of all maintenance actions available, see (4). The estimates are then used to select all seemingly beneficial maintenance actions (this is the leap). This despite that every maintenance action is evaluated singularly, neglecting the consequences of all the other actions. This approach does not warrant that a local optimum is reached and hence we proceed with more cautious "steps". I.e. we continue with a new simulation based on the chosen maintenance actions from the previous step/leap. As before, we evaluate all available maintenance actions, but here only select the most beneficial maintenance action (hence this is called a step). The steps and reliability calculations are then performed until no more improvements are found. The achieved optimal point is stored. One optimization cycle is then accomplished and the scale, $s$, is incremented, the calculation continues with a leap starting from the previous optimum. This is continued until there are no more scales to optimize for.

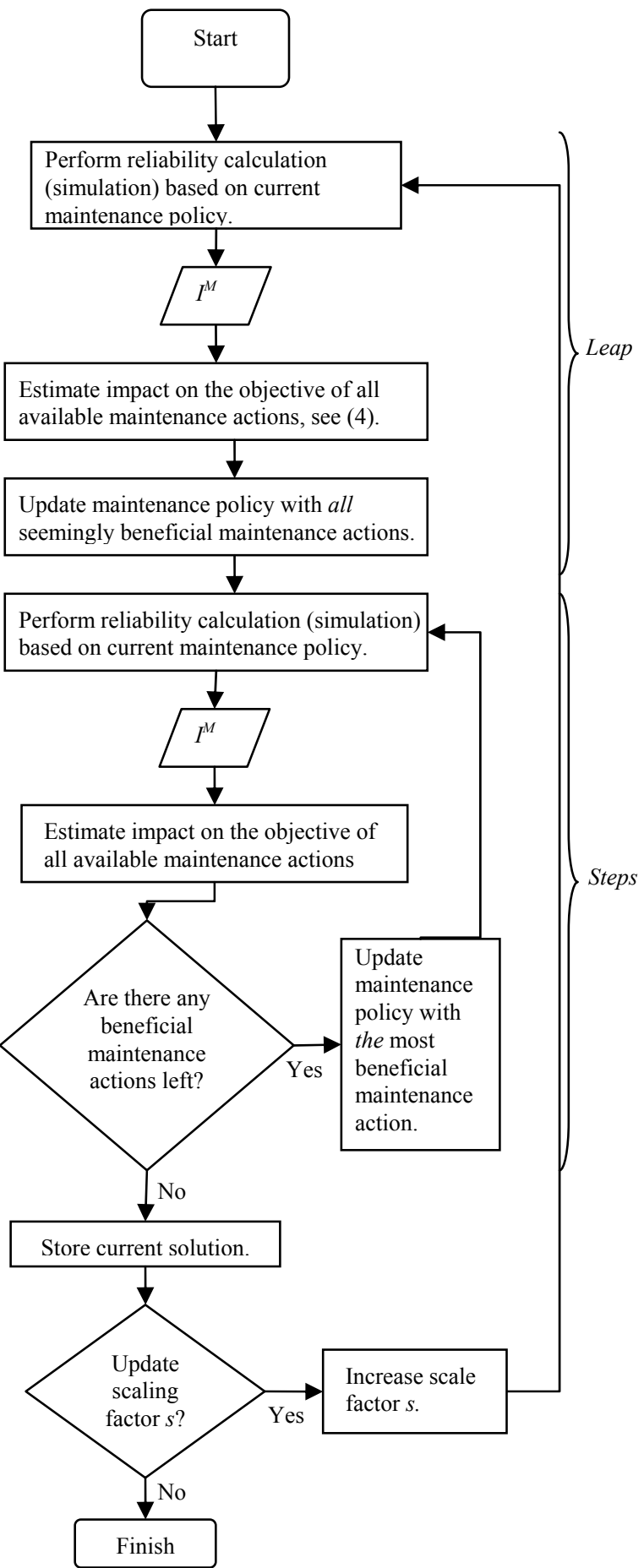

Fig 1. Flowchart for the optimization process. 
In both leap and step the following approximate evaluation of each individual maintenance alternative is made:

$$
P_{i, j} \approx \Delta \lambda_{i, j} C_{i}^{C M}+\Delta C_{i, j}^{P M}+s \Delta \lambda_{i, j} \frac{I_{i}^{M}}{\lambda_{i}}[€ / \mathrm{yr}, \text { action }]
$$

where $P_{i, j}$ is the expected change of the objective function if maintenance alternative $j$ is implemented for component $i, s$ is the scale factor of customer interruption costs, $\lambda$ is the component failure rate, $\Delta$ denotes the change from the current maintenance policy in the optimization process. $C_{i}^{C M}$ corresponds to the expected corrective maintenance cost that a failure of component $i$ incur for the DSO. $\Delta C^{P M}$ depends on the cost difference of introducing the studied maintenance action compared to the current policy. Equation (4) is approximate since the last term is an approximation on how the customer interruption cost will change. For each component in the leap the lowest value of $P$ is chosen for the next simulation. In the steps only one action is chosen, i.e. based on the lowest value, given that this value is below zero.

\section{E. Results and selection of optimum}

Given that the method has not found local optima. The suggested approach will deliver a number of optimal points, for an example see the case study below. These points will be located on the Pareto border and are all optimal from a specific view. Which solution that is selected by the decision maker depends on many factors such as the current status and behavior of the network. The different solutions provide the decision maker with important information on expected consequences of the different maintenance policies.

\section{CASE STUDY}

\section{A. Network, the Birka system}

The studied Birka system is located in the southern parts of Stockholm. The name is derived from the former Birka Energi $\mathrm{AB}$, of which the presented network was a part. Birka Energi is now a part of the Fortum consortium. The system, see Fig. 2 , which is thoroughly presented in [13] and [14], includes a $220 / 110 \mathrm{kV}$ station (Bredäng) and one $110 / 33 \mathrm{kV}, 33 / 11 \mathrm{kV}$ station (Liljeholmen). These two stations are connected with two parallel $110 \mathrm{kV}$ cables. From the Liljeholmen station there are two outgoing $33 \mathrm{kV}$ feeders, Högalid (HD) and Railway (SJ), there are also 32 outgoing $11 \mathrm{kV}$ feeders (LH11), here represented by one average set of components (28-35). The model includes 178 components, numbered 1-58, with 16 copies of component 28-35. The components are divided into five types, these are; circuit breakers (e.g. c2), cables (e.g. c5), transformers (e.g. c3), bus bars (e.g. c1) and fuses (c34). In the network, every component has a specific failure rate and repair rate. In total, this network serve approximately 38000 customers where the load point SJ consist of one customer, that is the railway. The load point LH11 represent one average load point of 32 actual outgoing feeders, which in total serve 14300 customers. The load point HD feeds approximately 23
400 customers [14]. In the model we have assumed piecewise constant failure and repair rates for every component, based on investigations of the network [14]. Note that the constant failure rates should be interpreted as population average values for the specific type of component population. Averages that can be modified by selected maintenance policies for the individual. Furthermore, the components are assumed to be independent.

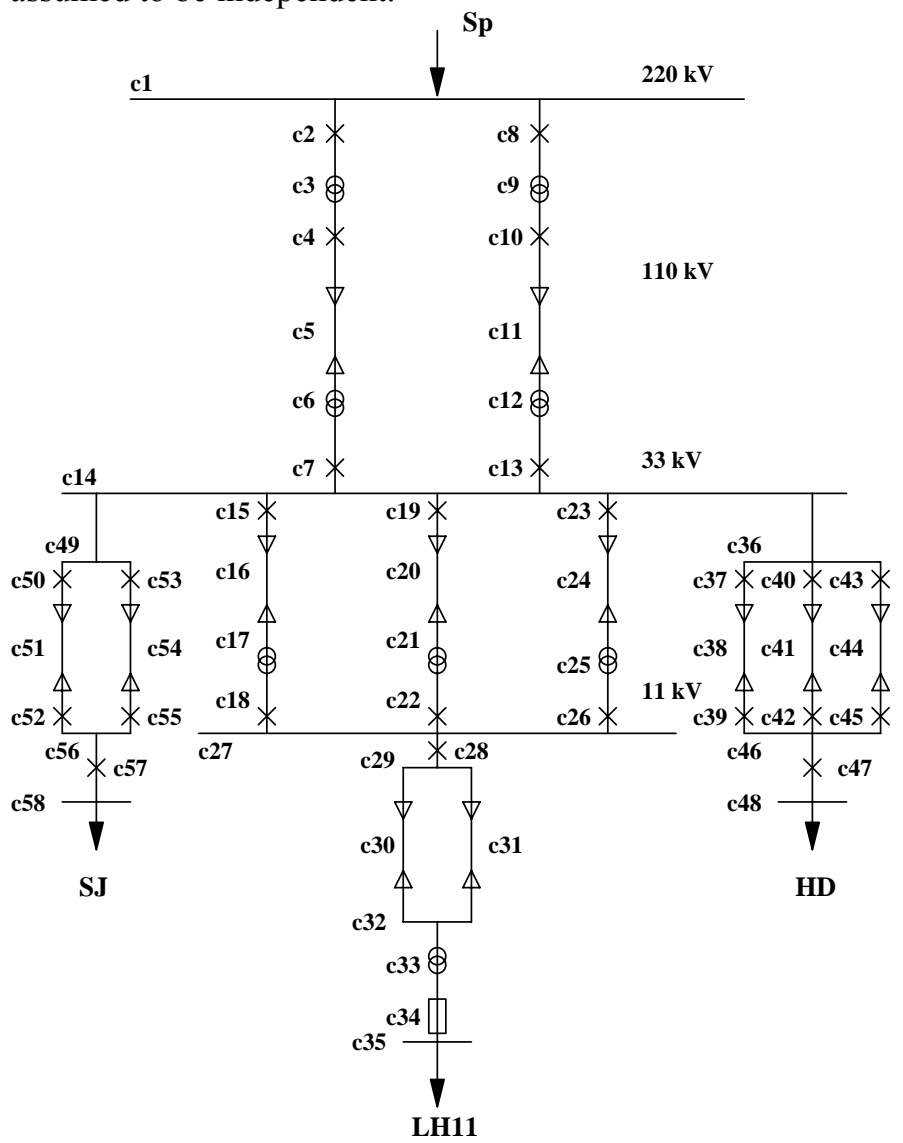

Fig 2. The studied system [13].

\section{B. Maintenance}

In this application study the maintenance actions are modeled on an aggregated level, i.e. if the components should be maintained as of today or if the preventive maintenance should be increased or decreased. This is modeled as there are three different preventive maintenance alternatives for each component in the network:

1. Keep current preventive maintenance level, average failure rate is assumed to remain unchanged, no change in cost for preventive and corrective maintenance.

2. Improve the preventive maintenance, the average failure rate is assumed to be halved for the studied component, the additional cost of this is one cost unit.

3. Decrease the preventive maintenance, the average failure rate is assumed to be doubled for the studied component, cost savings; one cost unit. 
Furthermore, it is assumed that the cost of one corrective maintenance action is $\beta$ cost units. In the basic case $\beta$ is put to 10 cost units. The cost of corrective maintenance is here chosen in relation to the maintenance effects in order to put incentives in the operator's maintenance budget to perform preventive maintenance. Studies have been performed for other values of $\beta$, see the sensitivity study section of this paper. The relationship between changes in failure rate and cost of corrective maintenance is displayed in (5).

$$
\Delta C^{C M}=\beta \sum_{\mathrm{i}}\left(\lambda_{i}^{\text {new }}-\lambda_{i}\right)
$$

where $i$ denotes component number. The change in preventive maintenance is calculated according to the formula in (6):

$$
\Delta C^{P M}=\operatorname{sum}(\text { incr maint })-\operatorname{sum}(\text { decr maint })
$$

where sum(incr maint) corresponds to the number of components with increased preventive maintenance actions and sum(decr maint) is the number of components with a decreased maintenance.

Note that the assumed maintenance alternatives in general "punish" relocation of maintenance resources in terms of total number of component failures. E.g. studying two components, both with the same initial failure rate, $\lambda$, and both being at alternative 1. By moving resources, i.e. putting one component to alternative 2 and the other to alternative 3 , this results in the sum $21 / 2 \lambda$ (compared to $2 \lambda$, before maintenance reallocation).

\section{Results}

The result of the optimization routine is a number of optimal points (solutions) which are all optimal from a specific point of scale. In Fig. 3 a number of optimal points are displayed, as well as the starting point (present situation). Note that since every optimization is built on results from a separate simulation, some of the optimal points are dominated by other optimal points. A point is dominated, when another point exist which is better in at least one criterion without being worse in any other criteria. The existence of these points is explained by the fact that every optimization is based on one or more (individual) simulations. In the previous work it has been seen that with more iterations in each simulation the number of dominated points decreases. In Table I more details are found for the presented solutions. The solutions 7-13 all dominate the "initial point". Even when considering SAIDI and SAIFI solutions 8-13 dominate the initial point, despite SAIDI and SAIFI not being directly included in the optimizations. The solutions 7-13 are probably more interesting than the other solutions obtained, since they do not make the situation worse for any of the two involved parties. This is however only true if we look at the total customer interruption cost. If we study every load point separately it can be seen in Table I that the interruption cost for node SJ is higher for solution 1-13 than for the starting point. One approach to this somewhat problematic situation might be to state that the solutions 7-13 constitute pareto improvements from a system perspective, which imply that we utilize our resources for the common good of the customers. Another approach might be to put constraints on the optimization, so that no customers will get worse reliability than of today, or penalize customer node interruption costs that are above today's level. If we want to investigate this issue further we need to split up the utilized customer objective into three new objectives, i.e. one measure for every load point. It is noteworthy that such approach most likely will be less efficient from a global perspective. In this paper we recognize the possibility of approaches that consider individual constraints on customer nodes. Nevertheless we continue the study with the focus on the common good, i.e. lowest total cost.

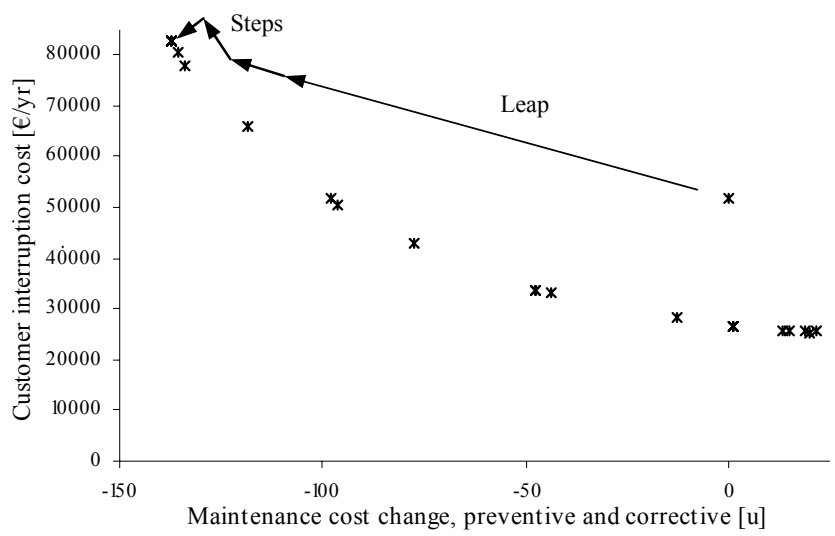

Fig. 3. The optimal solutions calculated. The $\mathrm{x}$-axis corresponds to changes in maintenance budget in comparison to today's budget. Note the starting point for the optimization (not an optimum), located at $(0,51$ 912). The arrows illustrate the optimization process to one optimum.

TABLE I

\begin{tabular}{|c|c|c|c|c|c|c|c|c|}
\hline lution & $\begin{array}{l}C_{L H 11}^{I C} \\
{[€ / \mathrm{yr}]}\end{array}$ & $\begin{array}{c}C_{H D}^{I C} \\
{[€ / \mathrm{yr}]}\end{array}$ & $\begin{array}{c}C_{S J}^{I C} \\
{[€ / \mathrm{yr}]}\end{array}$ & $\begin{array}{c}\sum \boldsymbol{C}^{\boldsymbol{I C}} \\
{[€ / \mathrm{yr}]}\end{array}$ & $\begin{array}{c}\boldsymbol{C}^{P M} \\
{[\text { units] }}\end{array}$ & $\begin{array}{c}\text { Mcomp. } \\
\text { failures } \\
{[\mathrm{f} / \mathrm{yr}]}\end{array}$ & $\begin{array}{c}\text { SAIFI } \\
{[\mathrm{int} / \mathrm{yr}]}\end{array}$ & $\begin{array}{l}\text { SAIDI } \\
{[\mathrm{h} / \mathrm{yr}]}\end{array}$ \\
\hline Org. & 47009 & 4283 & 620 & 51912 & $\mathbf{n} / \mathbf{a}$ & 4.01 & 0.144 & 0.271 \\
\hline $1,2,3$ & 73734 & 8049 & 1160 & 82943 & -144 & 4.66 & 0.211 & 0.451 \\
\hline 4 & 72103 & 7204 & 1046 & 80353 & -142 & 4.64 & 0.192 & 0.434 \\
\hline 5 & 70612 & 6302 & 909 & 77823 & -140 & 4.63 & 0.175 & 0.416 \\
\hline 6 & 58492 & 6361 & 917 & 65770 & -124 & 4.57 & 0.174 & 0.356 \\
\hline 7 & 46181 & 4726 & 788 & 51695 & -87 & 2.91 & 0.121 & 0.280 \\
\hline 8 & 45589 & 4225 & 725 & 50539 & -85 & 2.90 & 0.113 & 0.271 \\
\hline 9 & 39038 & 3398 & 727 & 43163 & -66 & 2.86 & 0.104 & 0.227 \\
\hline 10,11 & 29930 & 3147 & 694 & 33771 & -33 & 2.57 & 0.098 & 0.178 \\
\hline 12 & 29449 & 2934 & 698 & 33081 & -29 & 2.56 & 0.092 & 0.174 \\
\hline 13 & 24767 & 2929 & 701 & 28397 & 3 & 2.43 & 0.089 & 0.151 \\
\hline 14,15 & 23763 & 2495 & 515 & 26773 & 17 & 2.42 & 0.079 & 0.141 \\
\hline 16 & 23242 & 2232 & 409 & 25883 & 30 & 2.35 & 0.074 & 0.135 \\
\hline 17 & 23052 & 2213 & 344 & 25609 & 32 & 2.33 & 0.074 & 0.134 \\
\hline 18 & 23116 & 2091 & 347 & 25554 & 36 & 2.31 & 0.073 & 0.133 \\
\hline 19 & 23042 & 2095 & 307 & 25444 & 37 & 2.30 & 0.073 & 0.133 \\
\hline 20 & 23170 & 2068 & 306 & 25544 & 39 & 2.28 & 0.073 & 0.133 \\
\hline
\end{tabular}

OPTIMIZATION RESULTS

"Org." represent the non-optimized original solution, i.e. maintenance policy as of today. Data used in the optimization process are marked with bold caption. A number of solutions are identical; these are presented on the same row. $C^{P M}$ corresponds to the net change of preventive maintenance units. 


\section{General discussion of results}

If we study the optimization problem from the perspective of the DSO, one approach is to see how much we can decrease the maintenance budget, without decreasing the service to the customers. This is performed by identifying the solution with the nearest lower customer interruption cost compared to the solution of today $(0,51912)$. The maintenance cost difference between the starting point and the now reached optimal point gives us an estimate of today's maintenance policy inefficiency ${ }^{1}$. That is, how much it is possible to save on today's maintenance policy without reducing average customer service. This approach suggests solution number 7 for the case study, which significantly would reduce the cost of preventive maintenance. Likewise, we can perform this operation the other way around by going down from today's $(0,51192)$ solution to the pareto border in order to localize the point that given today's budget will give us the most satisfied customers. This approach suggests solution 13. According to Table I, this solution would with the utilized assumptions result in an almost halved customer interruption cost. While the preventive maintenance is increased for this solution the cost of corrective maintenance is lowered, resulting in a slightly lower maintenance cost, compared to today's maintenance cost.

\section{E. Results continued for one optimum (no. 10)}

In this section we study one of the reached optima in more detail, namely optimum number 10 , to exemplify a specific solution. Normally the DSO should choose a suitable solution. In this case we continue and assume that the DSO choose solution number 10 . This might be motivated by that this point has a suitable combination of lowered customer interruption costs and lowered maintenance cost, one additional advantage of this point is that the interruption cost for node 3 is relatively close to the starting value.

The resulting maintenance plan says that the preventive maintenance level should be increased for 56 components while decreased for 89 components and kept the same for 33 components. Fig. 4 presents an illustration of the suggested actions for optimum number 10 applied to the network.

\section{VALIDATION AND SENSITIVITY ANALYSIS}

\section{A. Validation of optimization method}

The presented problem has been approached with an additional tool, the Evolutionary Particle Swarm Optimization (EPSO) algorithm, developed at INESC Porto [15].

The EPSO algorithm can be described as a combination of evolutionary algorithms [16] and the particle swarm optimization algorithm (PSO) [17]. Somewhat simplified the EPSO works with a number of solutions (called particles) in the problem space. These particles have certain properties

\footnotetext{
${ }^{1}$ Since the studied example is based on partially fictive data, no definite conclusions can be drawn about the current management of the studied network.
}

such as position, inertia and memory. Evaluation of every particle is

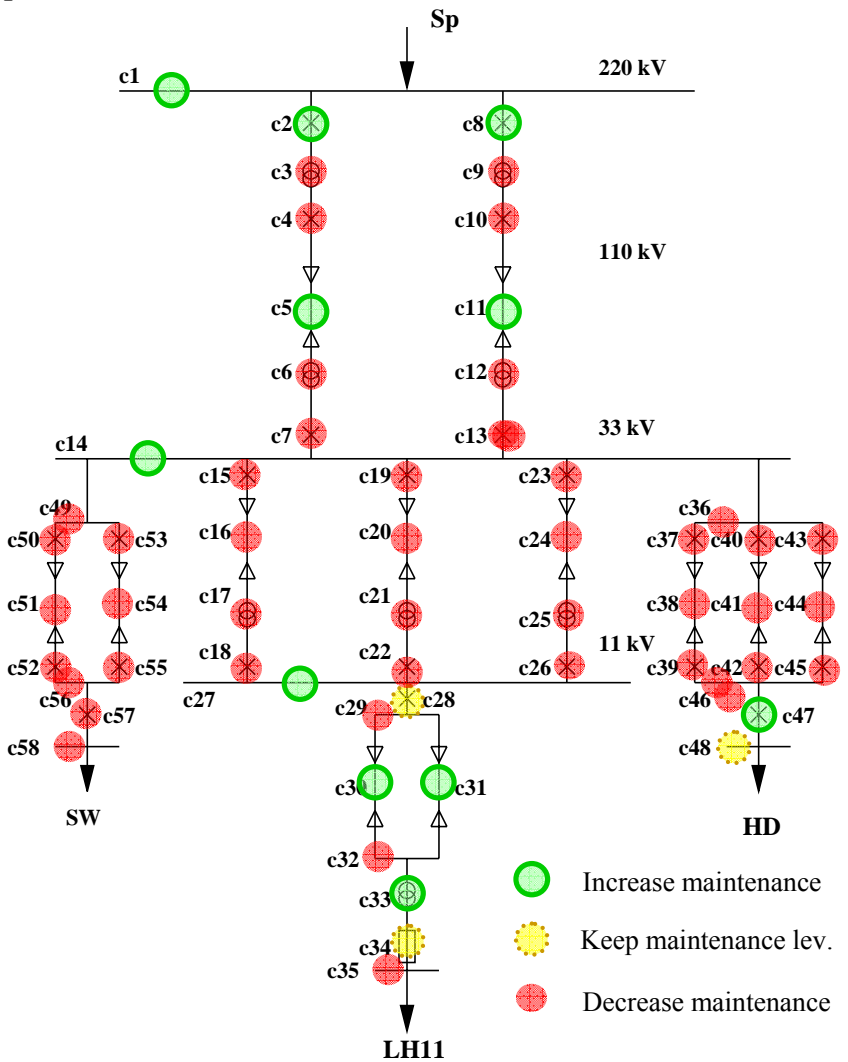

Fig. 4. Illustration of optimum number 10.

performed (calculation of objective function). Based on this evaluation a stochastic tournament is carried out, where good particles have higher chance to stay in the solver process than the less "good" particles. The particles "move" according to a combination of where the other particles are located (cooperation), inertia and memory (of the best point ever visited for the specific particle). The "surviving" particles from the tournament serve as a base for the generation of new particles replacing the ones sorted out. Furthermore, the method automatically mutates the weights of the different movement aspects (inertia, cooperation and memory). The mutation of the weight corresponds to an auto-tuning of parameters in a particle swarm optimization. Basically the difference between PSO and EPSO is that EPSO remove bad particles and spawns new ones and the mutation of movement weights.

The AGEBOM algorithm results were compared with the results from EPSO. Since EPSO to a certain degree utilize a random approach and investigate several solutions simultaneously the method is not as prone as AGEBOM to get stuck in local optima. Hence we investigated if the AGEBOM algorithm's solutions could be improved. No indications of improvements could be found, which indicates that the given problem formulation is without local optima that the AGEBOM get stuck in.

Since the AGEBOM algorithm require relatively few calls to the objective function and the objective function in this case 
is based on simulations the method becomes fast compared to the more intricate approach of the EPSO algorithm.

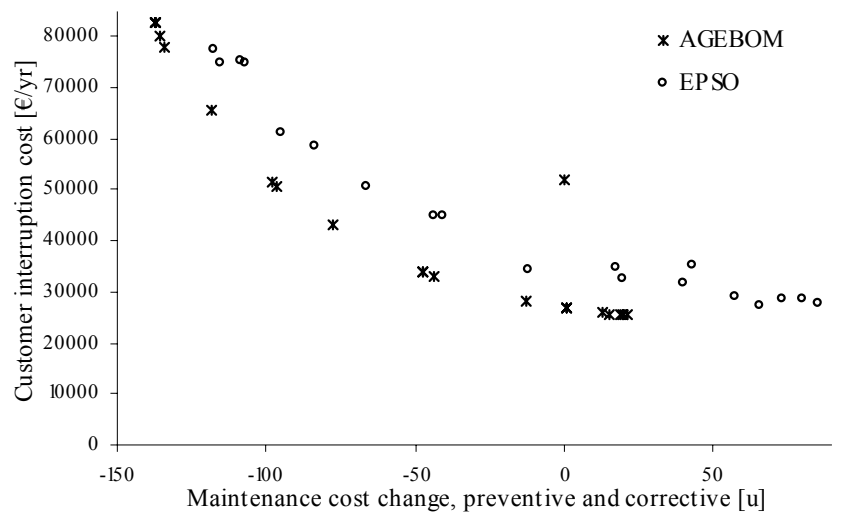

Fig. 5. Calculation results for EPSO (o) and AGEBOM (x). Note that the solution at $(0,51912)$ corresponds to today's maintenance policy.

The illustration above, Fig. 5, shows on the effectiveness of the developed AGEBOM algorithm compared to EPSO. The interpretation of this result is that the AGEBOM is well adapted to the studied problem. More complex problems may result in better relative performance by the EPSO algorithm.

\section{B. Sensitivity analysis}

One might argue that the above used assumptions on how the failure rate changes with changes in maintenance are somewhat optimistic. Hence, we look at an example of reduced efficiency in the allocation of preventive maintenance resources and a more expensive corrective maintenance. In this scenario the cost of corrective maintenance is tenfold compared to the first studied case $(\beta=100)$. Increased preventive maintenance results in only $10 \%$ reduction of the failure rate and decreased preventive maintenance results in $100 \%$ increased failure rate (as before).

Despite the effects of the changes, presented above, the process produce improvements, i.e. where we for example are capable of reducing the customer interruption costs by approximately one fifth while keeping today's maintenance budget. See Fig. 6 for an illustration of the results.

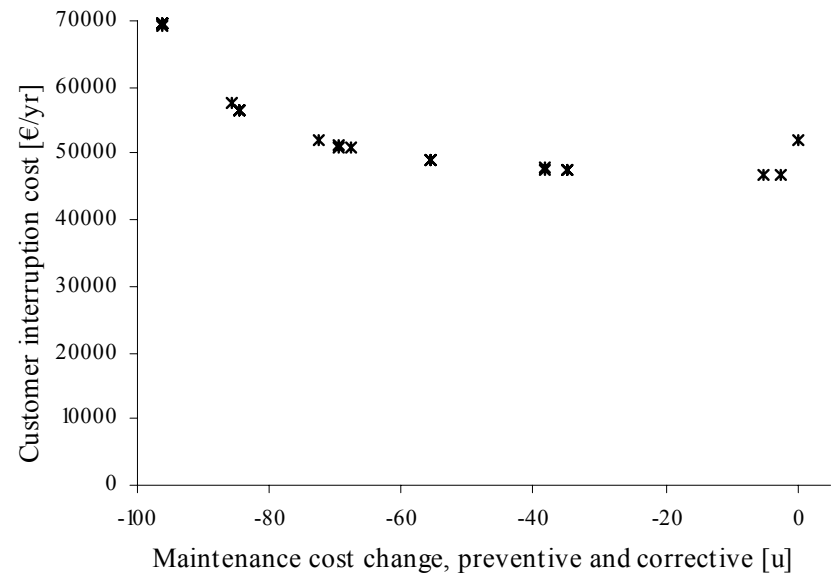

Fig. 6. Illustration of optimal points for expensive corrective maintenance and big losses in reallocation of maintenance resources. Note that the solution at $(0,51912)$ corresponds to the present maintenance policy.
It can be seen in this section that there still is a value of optimizing the maintenance despite large "penalties" in reallocating maintenance resources. The benefits do not become as significant as with the basic assumptions, but still there are benefits of the optimization. This is an effect of providing an existing solution (today's policy) with more alternatives that we optimize over. What actually is shown in this section is more of an illustration on how the results may vary within an actual outcome. And that there likely is value in introducing more maintenance policies for every component and to chose the right level of maintenance for each component with respect to network performance. For a more extensive sensitivity analysis, where for example only the reallocation of resources is penalized harder, see [18].

\section{Discussions \& PotentIAL IMPROVEMENTS}

\section{A. More parties}

This paper only studies the problem of maintenance from two perspectives, i.e. customers and DSOs. This is somewhat of a simplification since there are more parties involved in the process, such as regulatory authorities and third part maintenance providers. Regulatory issues can be accumulated within a widened definition of maintenance budget. For example penalties that the DSO has to pay because of to low reliability in one area and/or increased/decreased maximum allowed charge per kWh can be incorporated into the model. Third part maintenance providers may also complicate the optimization further, but are in this paper considered to perform specified policies and their impact is not further analyzed here.

\section{B. Network performance from customer view}

To use customer interruption costs as a performance measure may seem somewhat far-fetched but carries a number of advantages. Among those advantages is the possibility to incorporate both the effects of short power failures $(\mathrm{kW})$ and of longer interruptions $(\mathrm{kWh})$ in one measure. Additionally it is possible to include concepts of power quality and importance of different customers into a customer cost perspective. Furthermore, money is a measure that is possible to communicate throughout organizations and to people, all the way from the customers to the board of the DSO as well as regulating authorities. It is completely possible to establish values for interruption costs; this is performed in a number of studies e.g. [19] and [20]. Furthermore this aggregated measure is quite widely used in the process of network performance assessments. In Sweden [21] and Norway [22] the regulation of DSOs are partly based on interruption costs. Many companies use the customer interruption cost in investment planning. It is, however, perfectly possible to replace the proposed measure with another measure in the optimization, for example a weighted index based on SAIDI and SAIFI, as proposed in [3]. A modification in network performance measure requires a new component importance index, to replace (1). The conclusion being that the proposed customer network performance measure is adequate but that it 
may be replaced/modified due to situation specific reasons (e.g. a regulation based on SAIDI and SAIFI).

\section{CONCLUSION}

The results from the maintenance optimization are interesting. Despite a general decreased level of maintenance (lower total maintenance cost) a better network performance can be given to the customers. This is achieved by focusing the preventive maintenance on components with a high potential for improvement with respect to system performance. In the end this shows that there is value in introducing more maintenance policies for every component and to choose the right level of maintenance for every component. Here we have proposed a tool for this selection process.

The case study requires more data in order to demonstrate the true benefits of the maintenance policy optimization. Data on costs of corrective and preventive maintenance and estimates of how preventive maintenance affects the components are needed. However, in the less optimistic sensitivity analysis we still show on a possibility to improve the maintenance by use of the proposed method. The optimization method is based on time efficient heuristics, which include a risk of getting stuck in local optima in more complicated problem formulations. Here is the strength of using the EPSO algorithm in the optimization apparent. Although more costly in terms of computation time it is a tool that may prove very useful when optimizing more complicated problems.

\section{REFERENCES}

[1] Moubray, J. (1991). "Reliability-centered Maintenance" $2^{\text {nd }}$ edition. Butterworth-Heinermann, Oxford.

[2] Bertling, L., Allan, R., Eriksson, R., "A reliability-centered asset maintenance method for assessing the impact of maintenance in power distribution systems". IEEE Trans. Power Systems, vol 20, 2005.

[3] Li, F. and Brown, R. E., "A Cost-Effective Approach of Prioritizing Distribution Maintenance Based on System Reliability". IEEE Trans. Power Delivery, vol 19, 2004.

[4] Endrenyi, J., Anders, G., J., Bertling, L. and Kalinowski, B. "Comparison of Two Methods for Evaluating the effects of maintenance on component and system reliability". Proc. Probabilistic Methods Applied to Power Systems (PMAPS), Ames, Iowa, 2004.

[5] Zdrallek, M. "Reliability centred maintenance strategy for high-voltagenetworks". Proc. Probabilistic Methods Applied to Power Systems (PMAPS), Ames, Iowa, 2004.

[6] Neimane, V. (2001) "On development planning of electricity distribution networks", Doctoral Thesis, School of Electrical Engineering, KTH, Stockholm, Sweden, ISSN 1650-674x.

[7] Billinton, R. and Allan R. N., (1996). "Reliability evaluation of power systems". 2nd ed. Plenum Press, New York.

[8] Setréus, J. "Development of a simulation module for the reliability computer program RADPOW" MSc thesis 2006, School of Electrical Engineering, KTH, Stockholm, Sweden.

[9] Rausand, M. and Høyland, A. (2004) "System Reliability Theory", 2nd ed., John Wiley \& Sons, Hoboken, New Jersey, USA, ISBN: 0-47147133-X.

[10] Hilber, P. and Bertling, L. "A Method for Extracting Reliability Importance Indices from Reliability Simulations of Electrical Networks" Proc.15th Power Systems Computation Conference, PSCC 2005, Liege, Belgium.
[11] Keeney R., L. and Raiffa H. (1993): "Decisions with multiple objectives". Cambridge University press, Cambridge, United Kingdom, ISBN 0-521-43883-7.

[12] Hilber, P., (2005) "Component reliability importance indices for maintenance optimization of electrical networks", Licentiate thesis, School of Electrical Engineering, KTH, Stockholm, Sweden. ISBN 917178-055-6.

[13] Bertling, L. (2002) "Reliability Centred Maintenance for Electric Power Distribution Systems", Doctoral Thesis, School of Electrical Engineering, KTH, Stockholm, Sweden, ISBN 91-7283-345-9.

[14] Bertling, L., Eriksson R., Allan, R. N., Gustafsson, L. Å. and Åhlén, M. (2002). "Survey of causes of failures based on statistics and practice for improvements of preventive maintenance plans", Proc. $14^{\text {th }}$ Power Systems Computation Conference.

[15] Miranda, V., Fonseca, N. "EPSO-evolutionary particle swarm optimization, a new algorithm with applications in power systems", Proc. Transmission and Distribution Conference and Exhibition 2002> Asia Pacific, Volume: 2, pp 745- 750 IEEE/PES, 2002.

[16] Goldberg D. (1989). "Genetic Algorithms in Search, Optimization and Machine Learning", Harlow, England: Addison Wesley Longman, Inc.

[17] Kennedy, J. and Eberhart, R. "Particle swarm optimization. Neural Networks", Proc. IEEE International conference on neural networks, 1995 Perth, Australia.

[18] Hilber, P. "A Framework for Maintenance Policy Optimization of Electrical Networks, an Approximate Gradient Evaluation Bi-criteria Optimization Method". Internal report 2006, School of Electrical Engineering, KTH, Stockholm, Sweden.

[19] Tapper, M. et al. "Electric power interruption costs 2003", in Swedish. Swedenergy 2003.

[20] Billinton, R., convener. "Methods to consider customer interruption costs in power system analysis". 2001, Report 191, Task Force 38.06.01. Cigré.

[21] Gammelgård, M. "The Network Performance Assessment Model -A Review of the Swedish tool for Regulation", Proc. 5th Nordic Distribution and Asset Management Conference NORDAC 2002.

[22] G. H. Kjølle, A. T. Holen, K. Samdal, and G. Solum, "Adequate interruption cost assessment in a quality based regulation regime". Proc. IEEE PowerTech'01, vol. 3, Porto, Portugal, 2001.

Patrik Hilber (GS'02) was born in Stockholm 1975. In the year 2000 Patrik received the degree of M.Sc. in Systems Engineering and in 2005 the degree of Tech. Licentiate in Electrical Engineering both from KTH (Royal Institute of Technology), Stockholm, Sweden.

Since 2002 he is employed as a Ph.D. student within the RCAM research group at the School of Electrical Engineering, KTH, Stockholm, Sweden. Part of 2005 and 2006 he visited INESC Porto, Portugal, as a guest researcher. Patrik has industrial experience of optimization from previous employments, within the field of logistics optimization (1999-2002).

Vladimiro Miranda (M'90-SM'04-F'06) received his Licenciado, Ph.D. and Agregado degrees from the Faculty of Engineering of the University of Porto, Portugal (FEUP) in 1977, 1982 and 1991, all in Electrical Engineering. In 1981 he joined FEUP and currently holds the position of Professor Catedratico. He is also currently Director of INESC Porto. He has authored many papers and been responsible for many projects in areas related with the application of Computational Intelligence to Power Systems.

Manuel A. Matos (M'94) (El. Eng., Ph.D., Aggregation) was born in 1955 in Porto, Portugal. He is presently Full Professor at the Faculty of Engineering of the University of Porto, Portugal, and Manager of the Power Systems Unit of INESC Porto. He also collaborates with the Management School of the University of Porto. His research interests include fuzzy modeling of power systems, optimization and decision-aid methods. He is a member of IEEE.

Lina Bertling ( $\left.\mathrm{S}^{\prime} 98-\mathrm{M}^{\prime} 02\right)$ is Assistant Professor at KTH School of Electrical Engineering (the Royal Institute of Technology) in Stockholm Sweden. She received the Ph.D. in Electric power systems in 2002, and the M.Sc. degree in Systems engineering in 1997, both from KTH. She is the leader of the research group on reliability-centered asset management (RCAM), and the research program at the Swedish Centre of Excellence in 
Electric Power Systems (EKC2) on maintenance management. Her research interests are in; power system reliability modeling and maintenance planning and optimization. In 2006 she was the general chair of the 9th International conference on probabilistic methods applied to power systems (PMAPS) in Stockholm. 